\title{
Protonenresonanzspektren von Cyclopentadien, Methylcyclopentadien, Cycloheptatrien und Trimethylsiliciumdhlorid
}

\author{
Von Walter Strohmeier *, Erminio Lombardi und Richard M. Lemmon \\ Radiation Laboratory, University of California, Berkeley, USA. \\ (Z. Naturforschg. 14 a, 106-108 [1959]; eingegangen am 7. Oktober 1958)
}

\begin{abstract}
Es wurden mit Hilfe der kernmagnetischen Resonanzmethode die Protonenresonanzspektren von monomerem und dimerem Cyclopentadien und Methylcyclopentadien sowie von Cycloheptatrien und Trimethylsiliciumchlorid aufgenommen.

Die chemical shifts der Protonenresonanzen wurden unter Verwendung von Cyclohexan als „innerer Standard" bestimmt.

Monomeres Cyclopentadien besitzt 2 scharfe Resonanzlinien, deren chemical shift $\delta_{\mathrm{CH}}=+1,1 \mathrm{ppm}$ und $\delta_{\mathrm{CH}_{2}}=-2,5 \mathrm{ppm}$ ist. Die $4 \mathrm{CH}$-Protonen sind äquivalent.

Das Spektrum von monomerem Methylcyclopentadien zeigt Hyperfeinstruktur. 3 Gruppen von Resonanzlinien, entsprechend den $\mathrm{CH}-, \mathrm{CH}_{2}$ - und $\mathrm{CH}_{3}$-Protonen, wurden beobachtet.

Die Dimerisation des monomeren Cyclupentadiens und Methylcyclopentadiens wurde durch Aufnahme der Protonenresonanzspektren zeitlich verfolgt.

Aus dem Spektrum des Cycloheptatriens folgt, da $\beta$ die $\alpha-\beta$ - und $\gamma$-Protonen nicht äquivalent sind.

Im Trimethylsiliciumchlorid ist die chemical shift der Protonenresonanz $\delta_{\mathrm{CH}_{3}}=-4,8 \mathrm{ppm}$.

In Cyclohexan als Lösungsmittel war die chemical shift für Cyclopentadien und Methylcyclopentadien im Konzentrationsbereich $x=0,2-0,8$ ( $x=$ Molenbruch) von der Konzentration unabhängig.
\end{abstract}

\section{Methodik und Substanzen}

Die Protonenresonanzspektren wurden mit einem High Resolution NMR Spektrometer V 4300 B von Varian Associates (Palo Alto, California, USA.) bei $40 \mathrm{Mc}$ aufgenommen. Die chemical shift $\delta$ ist definitionsgemäß ${ }^{1}$

$$
\delta=10^{6}\left(H_{\mathrm{H}_{2} \mathrm{O}}-H_{\mathrm{Res}}\right) / H_{\mathrm{H}_{2} \mathrm{O}},
$$

wenn $H_{\mathrm{H} 2 \mathrm{O}}$ die magnetische Feldstärke ist, bei welcher die Protonenresonanz des Wassers auftritt, und $H_{\text {Res }}$ die Resonanzfeldstärke für die Protonen in der untersuchten Verbindung ist.

Zur Kalibrierung des magnetischen Feldes wurde Äthanol verwendet. Aus dem Abstand der $\mathrm{CH}_{3}$ - und $\mathrm{OH}-\mathrm{Frequenz}$ von $125 \mathrm{c} / \mathrm{s}$ (cycles/sec) bei $30 \mathrm{Mc}$ ergibt sich der $\mathrm{CH}_{3}-\mathrm{OH}$-Abstand der Resonanzlinien zu 4,2 ppm (s. Anm. ${ }^{2}$ ).

Zur Festlegung der chemical shift wurden die Substanzen in Cyclohexan gelöst und gemessen. Die Protonenresonanz der $\mathrm{CH}_{2}$-Gruppe des Cyclohexans wurde dabei als „innerer Standard“ verwendet. Für die chemical shift $\delta_{\mathrm{CH} 2}$ in $\mathrm{C}_{6} \mathrm{H}_{12}$ wurde $\delta_{\mathrm{CH} 2}=$

* Appointment, supported by the International Cooperation Administration under a program administered by the $\mathrm{Na}$ tional Academy of Sciences. Heimatadresse: Phys.-Chem. Institut der Universität Würzburg.

${ }^{1}$ L. H. Meyer, A. Seika u. H. S. Gutowsky, J. Amer. Chem. Soc. 75, 4567 [1953]. In der neueren Literatur wird $\delta$ jedoch meist in ppm (parts per million) ausgedrückt, daher der Faktor $10^{6}$ in obiger Formel, an Stelle von $10^{5}$ in der Originalliteratur.
- 3,9 ppm genommen ${ }^{1}$. Zur Festlegung der Protonenresonanz der reinen Verbindungen oder für Lösungen in Benzol wurde Cyclohexan als äußerer Standard verwendet. Die so erhaltenen Werte wurden auf den inneren Standard Cyclohexan empirisch umgerechnet.

Dimeres Cyclopentadien * : Dieses wurde zweimal bei $50 \mathrm{~mm}$ destilliert und die $82^{\circ}$. Fraktion aufgefangen, welche einen Schmelzpunkt von $33^{\circ} \mathrm{C}$ hatte ${ }^{3}$.

Monomeres Cyclopentadien: Dieses wurde durch thermische Spaltung des Dimeren dargestellt ${ }^{4}$. Am Kopf der Kolonne befand sich ein Rückflußkühler, in welchen Wasser von $43^{\circ}$ eingespeist wurde. Die Vorlage war auf $-80^{\circ}$ gehalten. Das Destillat wurde durch Gaschromatographie (Wilkens Aerograph) unter Verwendung von Paraffin-, Ucon-, Carbowax-, Silicon- und Triheptylamin-Substrat auf Reinheit geprüft. Es konnten keine Verunreinigungen nachgewiesen werden.

Dimeres Methylcyclopentadien *: Die Substanz wurde zweimal bei $30 \mathrm{~mm}$ destilliert und die $98^{\circ}$.Fraktion aufgefangen ${ }^{5}$.

Monomeres Methylcyclopentadien: Dargestellt ana$\log$ zu Cyclopentadien, nur wurde in den Rückflußkühler Wasser von $75^{\circ}$ eingespeist. Reinheitsprobe durch Gaschromatographie. Die Substanz erwies sich als einheitlich.

2 J. T. Arnold, Phys. Rev. 102, 136 [1956].

* Wir danken der Ethyl Corporation für die zur Verfügung gestellte Substanz.

${ }^{3}$ K. Uhrig, E. Lynch u. H. C. Becker, Ind. \& Eng. analyt. Edit. 18, 550 [1946]. - C. R. Gardner, Analyt. Chem. 20, 331 [1948].

${ }^{4}$ R. B. Moffet, Org. Synth. 32, 41 [1952].

${ }^{5}$ K. C. Edson, J. S. Powell u. E. L. Fisher, Ind. \& Eng. 40, 526 [1948]. 
Cycloheptatrien: Dargestellt aus Benzol und Diazomethan ${ }^{6}$. Die Substanz wurde durch Gaschromatographie unter Verwendung von Ucon-Substrat gereinigt.

Trimethylsiliciumchlorid: Verwendet wurde ein Präparat der Anderson Chemical Company (Weston, Michigan), welches durch Gaschromatographie von den enthaltenen Chlorwasserstoffspuren befreit wurde.

\section{Ergebnisse und Diskussion}

Die Protonenresonanzspektren von monomerem Cyclopentadien und Methylcyclopentadien, sowie von Cycloheptatrien und Trimethylsiliciumchlorid sind in Abb. 1 wiedergegeben.

Für Cyclopentadien wurde eine scharfe Resonanzlinie für die CH-Protonen mit $\delta_{\mathrm{CH}}=+1,1 \mathrm{ppm}$ und für die $\mathrm{CH}_{2}$-Protonen mit $\delta_{\mathrm{CH}_{2}}=-2,5$ ppm gefunden. Die 4 CH-Protonen sind, da das Spektrum nur eine scharfe Linie für die CH-Protonen aufweist, in bezug auf das am Kern angreifende magnetische Feld äquivalent. Unter den oben angegebenen Versuchsbedingungen konnte keine Hyperfeinstruktur nachgewiesen werden.

Das Protonenresonanzspektrum des Methylcyclopentadiens besteht aus 3 Gruppen von Linien, welche durch die $\mathrm{CH}$-, $\mathrm{CH}_{2}$ - und $\mathrm{CH}_{3}$-Protonen verursacht werden. Der Schwerpunkt der chemical shift ist für $\delta_{\mathrm{CH}} \sim+1,1$ ppm und $\delta_{\mathrm{CH}_{2}} \sim-2,5 \mathrm{ppm}$.

Da die einzelnen Linien der CH- und $\mathrm{CH}_{2}$-Resonanz zum Teil überlagert sind, kann zur Zeit keine Aussage gemacht werden, welche Linien durch Kernspin-Kopplung, second order-Effekte oder durch nichtäquivalente CH-Protonen erzeugt werden. Durch eine Aufnahme des Spektrums bei $60 \mathrm{Mc}$ könnte diese Zuordnung vielleicht durchgeführt werden. Die Möglichkeit, daß es sich bei dem untersuchten Methylcyclopentadien um eine Mischung von 1-Methylund 2-Methylcyclopentadien handelt, kann mit einiger Sicherheit ausgeschlossen werden, da es mit Hilfe der Gaschromatographie unter Verwendung von 5 verschiedenen Substraten nicht gelang, die Verbindung in ihre Isomeren aufzuspalten ${ }^{7}$. Es wurde nicht einmal die Andeutung einer "Schulter“ am Chromatogramm beobachtet.

Die Resonanz der $\mathrm{CH}_{3}$-Protonen des Methylcyclopentadiens lag bei $\delta_{\mathrm{CH}_{3}}=-3,3 \mathrm{ppm}$. Die Linie zeigte eine gerade noch erkennbare Struktur.

Das Protonenresonanzspektrum des Cyclohepta-

6 W. von E. Doering u. L. K. Knox, J. Amer. Chem. Soc. 75, 297 [1953].

7 Unabhängig von uns wurde früher bereits im Forschungslaboratorium der Ethyl-Corporation in Detroit versucht, das Methylcyclopentadien durch Gaschromatographie in die Iso-
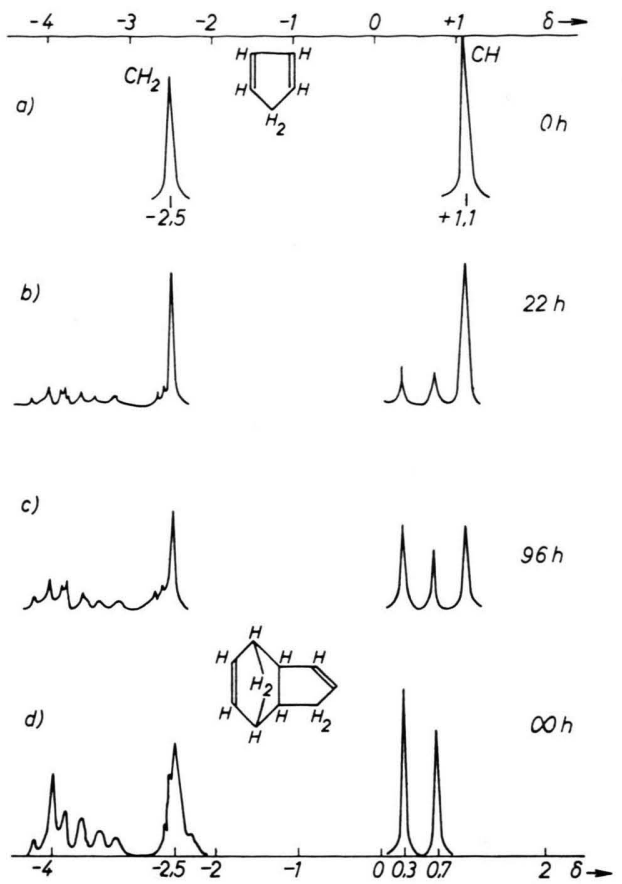

Abb. 1. Protonenresonanzspektrum von a) Cyclopentadien, b) Methylcyclopentadien, c) Cycloheptatrien und d) Trimethylsiliciumchlorid. Die chemical shift ist auf den inneren Standard Cyclohexan bezogen (mit $\delta_{\mathrm{CH}_{2}}$ in $\left.\mathrm{C}_{6} \mathrm{H}_{12}=-3,9 \mathrm{ppm}\right)$. Molenbruch $x$ der Verbindungen war $x=0,8$.

triens zeigt die Resonanz der $\mathrm{CH}_{2}$-Protonen bei $\delta_{\mathrm{CH} 2}=-3,2 \mathrm{ppm}$, während für die CH-Protonen mehrere Liniengruppen, welche zum Teil überlagert sind, gefunden wurden. Die 4 Resonanzlinien bei $\delta_{\mathrm{CH}}=-0,5 \mathrm{ppm}$ können den beiden Protonen in $\alpha$-Stellung zugeschrieben werden. Durch KernspinKopplung mit den Protonen der $\mathrm{CH}_{2}$-Gruppe und dem Proton in der $\beta$-Stellung wird die Resonanzlinie der $\alpha$-Protonen in 4 Linien aufgespalten. Das scharfe Triplett der $\mathrm{CH}_{2}$-Resonanz ist der Beweis, daß eine Kernspin-Kopplung zwischen den $\mathrm{CH}_{2}$ und den $\alpha$-Protonen stattfindet. Eine definitive $\mathrm{Zu}$ ordnung der restlichen Linien zu den $\beta$ - und $\gamma$-Protonen ist wegen ihrer Überlappung nicht möglich. Der Schwerpunkt der chemical shift der $\beta$ - und $\gamma$ Protonen liegt ungefähr in der Gegend der $\mathrm{CH}$ Resonanz des monomeren Cyclopentadiens und Methylcyclopentadiens.

Für Trimethylsiliciumchlorid wurde eine einzige

meren aufzuspalten, was ebenfalls nicht gelang. (Es konnte leider nicht in Erfahrung gebracht werden, ob es sich bei der verwendeten Substanz um 1 Methyl- oder 2 Methylcyclopentadien handelte.) Wir danken Herrn Direktor Dr. W. B. Ligetr für diese Privatmitteilung. 


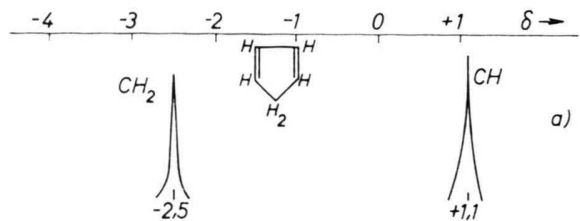

a)

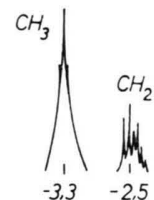

$\sqrt[H]{\mathrm{H}_{2}} \mathrm{CH}_{3}^{\mathrm{H}}$

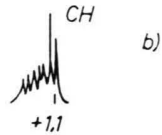

b)
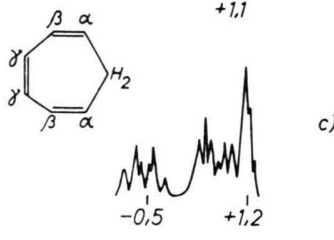

$\left(\mathrm{CH}_{3}\right)_{3} \mathrm{SiCl}$

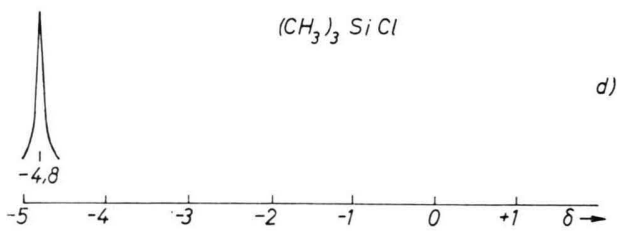

Abb. 2. Protonenresonanzspektrum von monomerem Cyclopentadien als Funktion der Zeit $\left(t=20^{\circ}\right)$; a) direkt nach der Herstellung, b) nach $22 \mathrm{~h}$, c) nach $96 \mathrm{~h}$, d) von dimerem Cyclopentadien.

scharfe Resonanzlinie mit $\delta_{\mathrm{CH}_{3}}=-4,8$ ppm gefunden. In Tetramethylsilicium ist die chemical shift 205 cycles bei $40 \mathrm{Mc}$ oder $\delta_{\mathrm{CH}_{3}}=-5,15 \mathrm{ppm}^{8}$ (für die reine Substanz). Unter dem Einfluß des elektronegativen Chloratoms werden im $\left(\mathrm{CH}_{3}\right)_{3}$ SiCl-Elektronen vom Silicium weggezogen, die chemical shielding der Protonen wird verringert, so daß die Resonanzlinie in Richtung abnehmender äußerer Feldstärke verschoben wird.

Die Resonanzspektren für die reinen Verbindungen waren identisch mit den Spektren in Cyclohexan- und Benzollösungen $(x=0,8 ; 0,45$ und 0,3$)$.

Die Protonenresonanzspektren von dimerem Cyclopentadien und Methylcyclopentadien sind in Abb. $2 \mathrm{~d}$ und $3 \mathrm{~d}$ wiedergegeben. Im dimeren Cyclopentadien wurden für die CH-Protonen 2 scharfe Resonanzlinien mit $\delta_{\mathrm{CH}}=+0,3$ und $+0,7$ ppm gefunden. Die CH-Protonen sind nicht mehr äquivalent wie in der monomeren Verbindung, was auch aus der Struktur des Dimeren folgt ${ }^{9}$. Das gleiche gilt für die $\mathrm{CH}_{2}$-Protonen. Allerdings ist die Resonanzlinie der einen $\mathrm{CH}_{2}$-Gruppe an der gleichen Stelle wie im Monomeren. Ähnlich liegen die Verhältnisse bei dimerem Methylcyclopentadien. Das Spektrum zeigt 3 Gruppen von Resonanzlinien, entsprechend den
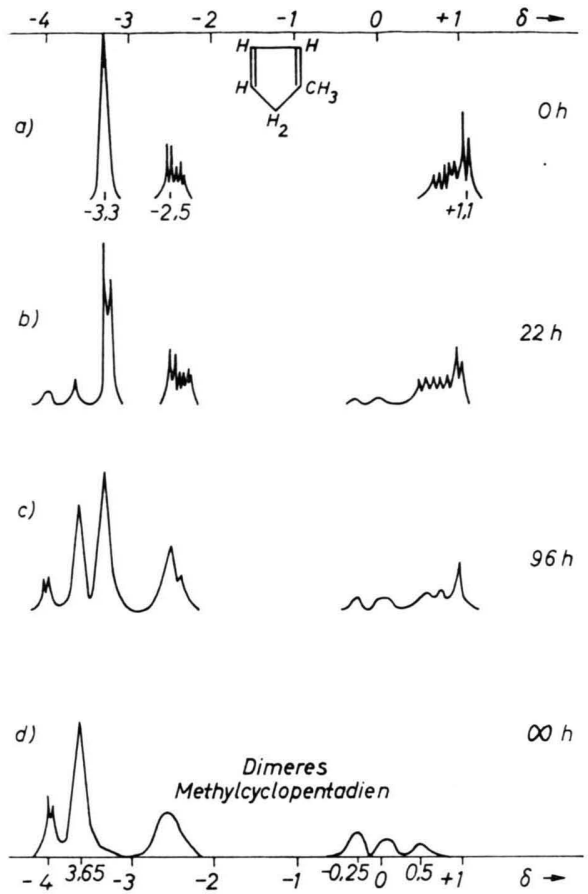

Abb. 3. Protonenresonanzspektrum von monomerem Methylcyclopentadien als Funktion der Zeit $\left(t=20^{\circ}\right)$; a) direkt nach der Herstellung, b) nach $22 \mathrm{~h}$, c) nach $96 \mathrm{~h}, \mathrm{~d}$ ) von dimerem Methylcyclopentadien.

$\mathrm{CH}$-, $\mathrm{CH}_{2}$ - und $\mathrm{CH}_{3}$-Protonen. Im Vergleich zum monomeren Methylcyclopentadien ist im Dimeren die Feinstruktur völlig verloren gegangen, und es wurden relativ breite Resonanzkurven beobachtet. Es ist wahrscheinlich, daß es sich beim dimeren Methylcyclopentadien um eine Mischung verschiedener Isomerer handelt.

Da sich Cyclopentadien und Methylcyclopentadien mit mäßiger Geschwindigkeit dimerisiert ${ }^{10}$, wurde das Protonenresonanzspektrum als Funktion der Zeit nach $22 \mathrm{~h}$ bzw. $96 \mathrm{~h}$ aufgenommen. Wie Abb. 2 und 3 zeigen, kann die kernmagnetische Resonanz mit Vorteil zur Untersuchung der Dimerisation und somit auch der Polymerisation verwendet werden. Die Protonenresonanzspektren von dimerem Cyclopentadien und Methylcyclopentadien, erhalten durch Dimerisation der Monomeren bei Zimmertemperatur, waren identisch mit den Spektren der Dimeren, welche durch Destillation bei $50 \mathrm{~mm}$ bzw. $30 \mathrm{~mm}$ Druck (siehe unter Substanzen) aus den Ausgangsprodukten erhalten wurden.

8 A. L. Allred u. E. G. Rochow, J. Inorg. u. Nucl. Chem. 5, 269 [1958].

9 Ph. J. Wilson u. J. H. Wells, Chem. Rev. 34, 1 [1944].

10 K. C. Edson, J. S. Powell u. E. L. Fisher, Ind. and Eng. 40, 1526 [1948]. 